\section{Which antiretroviral regimen is associated with higher adherence in Brazil? A comparison of single, multi, and dolutegravir-based regimens}

\author{
Qual esquema antirretroviral está associado à \\ maior adesão no Brasil? Uma comparação dos \\ esquemas de comprimido único, múltiplos \\ comprimidos e com dolutegravir
}

\section{¿Qué tratamiento antirretroviral está asociado con adherencia más alta en Brasil? Una comparación de tratamientos únicos, múltiples y basados en el dolutegravir}

Tarsilla Spezialli Cardoso 1 Juliana de Oliveira Costa 1 Edna Afonso Reis 1

Micheline Rosa Silveira 1

Palmira de Fátima Bonolo 1

Simone Furtado dos Santos 1

Maria das Graças Braga Ceccato 1

\begin{abstract}
We evaluated adherence to highly active antiretroviral therapy (HAART) and its associated factors according to the type of regimen in patients initiating treatment in Belo Horizonte, Minas Gerais State, Brazil. We measured adherence using the eight items Morisky Therapeutic Adhesion Scale (MMAS-8) and compared the use of "backbone" tenofovir/lamivudine plus efavirenz one tablet once-daily (STR) or dolutegravir in multi-tablet oncedaily (MTR-DTG), or other multi-tablet regimens (MTR-other). We conducted a multivariate logistic regression analysis to address factors associated with adherence. A total of 393 patients were included, 254 used STR, 106 MTRDTG, and 33 MTR-other. The overall adhesion rate was 44.8\% (95\% CI: 39.4; 50.1), 50\% for MTR-DTG, 43.3\% for STR and 39.4\% for MTR-other. Multivariate analysis showed a higher chance of adherence among patients using MTR-DTG, those who received and understood counseling about their treatment and with a higher quality of life. Prior use of illicit drugs in the lifetime was associated with poorer adherence. Overall adherence was low, highlighting the need for strategies focusing on counseling about medicines and substance use. Pill burden was not an issue for patients using MTR-DTG once-daily, who achieved better results.
\end{abstract}

HIV; Anti-retroviral Agents; Medication Adherence; Self Report

\section{Correspondence}

J. O. Costa

Universidade Federal de Minas Gerais.

Av. Prof. Alfredo Balena 190, Belo Horizonte, MG 30130-100, Brasil.

julianaoc@far.grad.ufmg.br

1 Universidade Federal de Minas Gerais, Belo Horizonte, Brasil 


\section{Introduction}

The therapeutic success of highly active antiretroviral therapy (HAART) is assessed by viral suppression and its achievement requires high levels of HAART adherence, generally 95\% 1,2. Lower levels of adherence may lead to therapeutic failure, viral mutations selection and the development of resistance to medicines 3,4 .

Adherence is a worldwide challenge and is associated with several factors related to the individual, to the medicines and to the health services. Major difficulties with HAART occur at the beginning of the treatment when the main factors affecting adherence are adverse reactions, number of tablets administered, level of schooling, the presence of AIDS symptoms, among others directly influencing the individual's routine 5,6 .

Simplification of HAART is a worldwide trend as a strategy to increase the adherence and include the use of single tablet regimens (STR) consisting of two or more drugs in a single tablet, the use of single daily regimens, and the use of medicines with lower toxicity ${ }^{7}$.

Previous studies have reported greater adherence to HAART for patients using STR containing tenofovir (TDF), emtricitabine (FTC) and efavirenz (EFV) 3,7,8. In Brazil, the generic STR is available to the population since 2015, however, it contains a different formulation: TDF, lamivudine (3TC) and EFV 4. In 2017, dolutegravir (DTG) was included in the first-line regimen for individuals initiating HAART, replacing EFV 4. This regimen includes one tablet containing TDF/3TC plus one DTG tablet, administered once-daily. However, the impact of different administration regimens in the adherence to HAART is still unknown.

The scarcity of observational studies in HAART-naïve individuals specifically evaluating the STR containing TDF/3TC/EFV or the TDF/3TC + DTG regimen, associated to the updating of the Brazilian guidelines, highlights the need to evaluate the adherence to the regimens available to the population.

Brazil has been recognized for its strong response to the HIV epidemic. The Federal government has provided since the early 1990s generic versions of antiretroviral drugs, and, it started providing in 2013 public antiretroviral treatment to all HIV-adults seeking care, regardless of the stage of HIV they were facing. Despite of the struggle for sustainable financing of the Brazilian Unified National Health System (SUS), Brazil's national HIV and AIDS response is integrated into the country's Health Strategic Plan. The approach to HIV prevention involves promoting and improving access to HIV testing, immediate treatment for those testing positive regardless of CD4 count, and the provision of pre- and post-exposure prophylaxis 4.

Primary care in SUS expanded its services to $64 \%$ of the national population in addition to the other HIV/AIDS public services, 655 reference services, 25 laboratories for HIV-1 diagnostic, 12 services to lipodystrophy treatment, and, 101 Centers for Testing and Counseling 4.

Accordingly, we aimed to evaluate the adherence to first-line antiretroviral regimens and associated factors among individuals initiating treatment in Southeast Brazil to contribute in developing target strategies to improve patient's outcomes.

\section{Methods}

This is a baseline cross-sectional analysis of the ECOART cohort study conducted in Belo Horizonte, capital of Minas Gerais State, Brazil. We evaluated people living with HIV with at least seven and maximum 180 days of HAART usage, selected by non-randomized sampling and identified through their register on the Medication Logistics Control System (SICLOM).

Patients were recruited from September 2015 to August 2017 in three public reference services in outpatient HIV care covering about $80 \%$ of people living with HIV in the city, according to registers from the SICLOM. Service I belongs to a large hospital of the public network of Minas Gerais that provides specialized assistance with interdisciplinary care and dispensing of medication to the patient. Service II is a specialized municipal outpatient assistance and also a testing and counseling center that provides comprehensive and interdisciplinary treatment and dispensing of medicines. 
Service III is also a municipal reference, and provides interdisciplinary assistance, integral and dispensing of medicines.

Inclusion criteria were individuals aged $13+$ years old, attending one of the three reference services during the recruitment period, who had a minimum autonomy to answer the interview, agreed to participate in the study and signed the consent form. We obtained data through face-to-face interviews with eligible patients and complemented with national data from the Laboratory Test Control System (SISCEL) and SICLOM electronic systems from the Brazilian Ministry of Health.

The research was conducted in accordance with the Resolution n. 466/2012 of the Brazilian National Health Council. All data collected were kept confidential and the identification of individuals was preserved. For the accomplishment of the research, we obtained the approval of the Ethics Research Committee of Minas Gerais Federal University (UFMG; CAAE-31192914.3.0000.5149) and of the participating centers.

\section{Events and explanatory variables}

Adherence (the dependent variable) was measured by self-report using the eight items Morisky Therapeutic Adhesion Scale (MMAS-8) 9,10,11 validated in Brazil 12. Scores ranged from 0 to 8 points and patients who obtained 8 points (100\%) were considered adherents. Independent variables were: (i) sociodemographic: sex, age, schooling level, own income, employment status, economic class, marital status; (ii) behavioral and habits of life: alcohol use, cigarette smoking, illicit drugs use in lifetime, condoms usage in the last month, risk/exposure category; (iii) clinical: AIDS-defining illness, signs and symptoms of anxiety and depression, self-reported comorbidities or coinfections; (iv) HAARTrelated: type of regimen, adverse reactions, number of adverse reactions, self-perception about the difficulty of treatment, time on HAART; (v) laboratory tests: CD4+ T lymphocyte count (LT-CD4+) and viral load at the beginning of HAART usage; (vi) service-related: health care unit and counseling of health professionals about HAART and (vii) quality of life.

The economy class classification was evaluated according to the Brazilian Association of Research Companies (ABEP) 13. Data for concurrent AIDS-defining illness was obtained from patient's clinical chart and classified according to the Centers for Disease Control and Prevention definition 14. Signs and symptoms of anxiety and depression were evaluated by the Hospital Anxiety and Depression Scale 15,16, with scores ranging from 0 to 14 , and a cut off of $\geq 8$ points for both anxiety and depression.

The HAART regimen was classified as STR (TDF/3TC/EFV one tablet once-daily), MTR-DTG once-daily (TDF/3TC + DTG once-daily) or other multiple tablet regimens (MTR-other), corresponding to all other multi-tablet multi-dose HAART regimens.

Self-perception about difficulty with treatment was measured by the following question: "Based on your experience with antiretroviral drugs so far, how would you rate your treatment on a daily basis?". Valid options of response were dichotomized as "difficult" (very difficult, difficult, medium) or "easy" (easy, very easy).

LT-CD4+ and viral load at the beginning of the treatment were retrieved from SISCEL with a tolerance of three months. Quality of life was assessed by the WHOQOL-HIV BREF instrument, with scores ranging from 0 to 20 for each one of the six domains 17,18 .

The interviews were typed into Epi Info 3.5.4 software (https://www.cdc.gov/epiinfo/index.html) and $10 \%$ of the sample was retyped for quality control, achieving a perfect inter-examiner agreement $($ kappa statistic $=0.95) 19$.

\section{Statistical analysis}

For analytic purposes, patients were compared according to the HAART regimen in use at the time of the interview: STR, MTR-DTG or MTR-other. A descriptive analysis was carried out followed by a bivariate analysis, stratified by type of regimen. The individual association of each categorical variable with adhesion was assessed by Pearson's chi-square test or Fischer's exact test, when appropriate. For continuous variables, the t-test was used. 
Multivariate analysis was performed through a logistic regression model and values were presented by odds ratio (OR) with their respective 95\% confidence intervals (95\%CI). Variables with a $\mathrm{p}$-value $\leq 0.20$ in the bivariate analysis were selected to enter the initial multivariate model. The backward stepwise method was conducted until the final model contained only relevant variables $(\mathrm{p} \leq 0,05$ or with theoretical relevance). The suitability of the model was assessed by the Hosmer-Lemeshow test and by the area under the Receiver Operating Characteristics (ROC) curve, with p-values $>0.05$ indicating a good fit of the model in the first test and values $>0.7$ in the second one.

Statistical analyses were performed using the SPSS v. 24 (https://www.ibm.com/) at a significance level of 5\% and forest plots were developed using the R (http://www.r-project.org).

\section{Results}

Among 460 eligible patients identified, 427 agreed to participate in the ECOART study. Of 427 persons, 31 were excluded due to lack of data on adherence and 3 because they had less than seven days of treatment initiation totalizing 393 (85\%) in the current analysis. There were no statistically significant differences between participants and non-participants regarding sex, age or race.

Most of the participants were male (82.4\%) and the main risk/exposure category corresponded to men who have sex with men - MSM (52.1\%). The majority was single, divorced or widowed (79.6\%) and was working at the time of the interview (61.3\%). Approximately half were under 33 years old (51.1\%), belonged to the economic class C (47.1\%) and 38.4\% had 10 to 12 years of schooling. A high proportion of patients consumed alcohol (79.6\%) and used illicit drugs in the lifetime (49.7\%). AIDSdefining illness occurred on $20 \%$ of participants and comorbidities were reported by $19.8 \%$ and $21.4 \%$ had signs and symptoms of depression and $31.3 \%$ of anxiety (Table 1 ).

Most patients used a therapeutic regimen containing 2 NRTIs + 1 NNRTI (66.7\%), being TDF, 3TC, EFV the most common. STR was used by 254 (64.6\%) patients, 106 (27\%) used MTR-DTG regimen and 33 (8.4\%) used MTR-other. Approximately 70.3\% considered the treatment easy and $54.7 \%$ were on HAART for more than 60 days at the time of the interview. Of the total number of patients, $85.9 \%$ had at least one and $72.6 \%$ up to five adverse reactions.

Initial LT-CD4+ count $\leq 200$ cells $/ \mathrm{mm}^{3}$ was observed for $27 \%$ of patients and $26.5 \%$ started HAART with viral load greater than 100,000 copies/mL. Most patients (41.7\%) attended service II and approximately $12 \%$ did not receive or did not understand the health professional counseling about HAART. Mean quality of life was higher than 14.0 in all domains, with the lowest value observed in the environment domain $(14.32 ; \mathrm{SD}=2.40)$ and the highest in the physical domain $(15.44 ; \mathrm{SD}=3.09)$ (Table 1).

\section{Adherence assessment}

Table 2 shows patient's responses to individual questions of the MMAS- 8 , where $97.7 \%$ of the patients did not take their HAART medicines because they felt better and thought the disease was under control (item 6). There was a higher frequency of responses indicating adherence among the MTRDTG group (five items) and a lower frequency in the MTR-other group (only one item). The overall prevalence of adherence was $44.8 \%(n=176)$ and MTR-DTG group presented the highest percentage of adherent individuals (50\%), followed by the STR group (43.3\%) and the MTR-other group (39.4\%). However, there was no statistically significant difference between adherence to HAART among the groups and the three participanting services $(\mathrm{p}=0.81)$.

The adherence to HAART in the overall population ranged from $28.6 \%$ to $61.8 \%$ according to their characteristics. Lower point estimates were observed for patients with signs and symptoms of depression (28.6\%), patients who did not receive or understand health professional counseling about HAART (31.3\%), patients who perceived their treatment as difficult (31.9\%), patients reporting more than five adverse reactions (32.7\%), those using 2 NRTI + 2 PI (33.3\%) and who had used illicit drugs in the lifetime (37.4\%). Higher adherence estimates were observed for individuals who did not have any adverse reaction (61.8\%), were married or in a stable union (52.5\%), who did not use illicit drugs in the lifetime (52.3\%), aged 33 years or more (51\%) and with $13+$ years of schooling (47.6\%) (Table 3 ). 
Table 1

Characteristics of individuals living with HIV, according to the antiretroviral regimen used. Belo Horizonte, Minas Gerais State, Brazil, 2017 ( $\mathrm{n}=393$ ).

\begin{tabular}{|c|c|c|c|c|c|}
\hline Characteristics & Global $(n=393)$ & $\operatorname{STR}(n=254)$ & MTR-DTG (n = 106) & MTR-other $(n=33)$ & p-value \\
\hline \multicolumn{6}{|l|}{ Sociodemographic } \\
\hline Sex (male) & $323(82.4)$ & 209 (82.3) & $90(85.7)$ & $24(72.7)$ & 0.23 \\
\hline Age (> 33 years) & $192(48.9)$ & $117(46.1)$ & $50(47.2)$ & $25(75.8)$ & $0.01 *$ \\
\hline Schooling level (years) & & & & & 0.09 * \\
\hline$\leq 9$ & $95(24.2)$ & $61(24.0)$ & $19(17.9)$ & $15(45.5)$ & \\
\hline $10-12$ & $151(38.4)$ & 104 (40.9) & $37(34.9)$ & $10(30.3)$ & \\
\hline $13+$ & $147(37.4)$ & $89(35.0)$ & $50(47.2)$ & $8(24.2)$ & \\
\hline Own income in the last 6 months (yes) & 324 (82.7) & $211(83.4)$ & $88(83.0)$ & $25(75.8)$ & 0.55 \\
\hline Employment status (working) & $241(61.3)$ & $165(65.0)$ & $62(58.5)$ & $14(42.4)$ & $0.03 *$ \\
\hline Economic class & & & & & $0.02 *$ \\
\hline$A-B$ & $140(36.5)$ & $95(38.5)$ & $38(35.8)$ & $7(22.6)$ & \\
\hline C & $181(47.1)$ & $109(44.1)$ & $58(54.7)$ & $14(45.2)$ & \\
\hline$D-E$ & $63(16.4)$ & $43(17.4)$ & $10(9.4)$ & $10(32.3)$ & \\
\hline Marital status (married/stable union) & $80(20.4)$ & $51(20.1)$ & $17(16.0)$ & $12(36.4)$ & 0.04 * \\
\hline \multicolumn{6}{|l|}{ Behavioral and habits of life } \\
\hline Ever used alcohol (yes) & $313(79.6)$ & 207 (81.5) & $82(77.4)$ & $24(72.7)$ & 0.40 \\
\hline Currently smoker (yes) & $102(26.0)$ & $66(26.0)$ & $29(27.4)$ & $7(21.2)$ & 0.78 \\
\hline Ever used any illicit drug (yes) & 195 (49.7) & $123(48.4)$ & $60(56.6)$ & $12(37.5)$ & 0.13 \\
\hline $\begin{array}{l}\text { Condom use in all sexual intercourses in the last } \\
\text { month (yes) }\end{array}$ & $222(70.3)$ & $141(67.1)$ & $64(76.2)$ & $17(77.3)$ & 0.23 \\
\hline Risk/Exposure category (MSM) & $198(52.1)$ & $134(54.3)$ & $52(51.5)$ & $12(37.5)$ & 0.20 \\
\hline \multicolumn{6}{|l|}{ Clinical } \\
\hline AIDS defining illness (AIDS) & $76(20.0)$ & $57(23.1)$ & $6(5.9)$ & $13(40.6)$ & $<0.01$ * \\
\hline Anxiety (yes) & $123(31.3)$ & $81(31.9)$ & $28(26.4)$ & $14(42.4)$ & 0.21 \\
\hline Depression (yes) & $84(21.4)$ & $59(23.2)$ & $15(14.2)$ & $10(33.3)$ & 0.07 \\
\hline Comorbidities (yes) & $78(19.8)$ & $51(20.1)$ & $13(12.3)$ & $14(42.4)$ & $<0.01 *$ \\
\hline Coinfections (yes) & $28(7.2)$ & $22(8.7)$ & $2(1.9)$ & $4(12.1)$ & $0.04 *$ \\
\hline \multicolumn{6}{|l|}{ HAART-related } \\
\hline Classical therapeutic regimen & & & & & - \\
\hline $2 \mathrm{NRTI}+1 \mathrm{NNRTI}$ & $262(66.7)$ & $254(100.0)$ & - & $8(24.2)$ & \\
\hline $2 \mathrm{NRTI}+1 \mathrm{IIN}$ & $110(28.0)$ & - & $106(100.0)$ & $4(12.1)$ & \\
\hline $2 \mathrm{NRTI}+2 \mathrm{PI}$ & $21(5.3)$ & - & - & $21(63.6)$ & \\
\hline Adverse reactions (yes) & 335 (85.9) & $226(89.7)$ & $81(76.4)$ & $28(87.5)$ & $<0.01$ * \\
\hline Number of adverse reactions (>5) & $107(27.4)$ & $85(33.7)$ & $10(9.4)$ & $12(37.5)$ & $<0.01 *$ \\
\hline Self-perception of treatment difficulty (easy) & $275(70.3)$ & $173(68.7)$ & $87(82.1)$ & $15(45.5)$ & $<0.01$ * \\
\hline Time since treatment initiation (> 60 days) & $214(54.7)$ & $152(60.1)$ & $38(35.8)$ & $24(75.0)$ & $<0.01 *$ \\
\hline \multicolumn{6}{|l|}{ Laboratory tests (before HAART) } \\
\hline CD4+ T lymphocyte count & $88(27.0)$ & $63(30.0)$ & $14(15.6)$ & $11(42.3)$ & $0.01 *$ \\
\hline \multicolumn{6}{|l|}{$\left(\leq 200\right.$ cells $\left./ \mathrm{mm}^{3}\right)$} \\
\hline Viral load (> 100,000 copies/mL) & $87(26.5)$ & $60(28.2)$ & $15(17.2)$ & $12(42.9)$ & $0.02 *$ \\
\hline \multicolumn{6}{|l|}{ Health service-related } \\
\hline Healthcare facility & & & & & $<0.01 *$ \\
\hline 1 & 145 (36.9) & $125(49.2)$ & $0(0.0)$ & $20(60.6)$ & \\
\hline II & $164(41.7)$ & $106(41.7)$ & $47(44.3)$ & $11(33.3)$ & \\
\hline III & $84(21.4)$ & $23(9.1)$ & $59(55.7)$ & $2(6.1)$ & \\
\hline Receiving and understanding HAART counseling (Yes) & $343(87.7)$ & $222(88.1)$ & $93(87.7)$ & $28(84.8)$ & 0.87 \\
\hline \multicolumn{6}{|l|}{ WHOQOL-HIV BREF domains [Mean (SD)] } \\
\hline Physical & $15.44(3.09)$ & $15.32(3.04)$ & $16.12(2.63)$ & $14.18(4.18)$ & $<0.01$ * \\
\hline Psychological & $14.98(2.83)$ & $14.94(2.92)$ & $15.43(2.27)$ & $13.82(3.46)$ & 0.02 * \\
\hline Independence & $15.43(2.75)$ & $15.47(2.78)$ & $15.66(2.37)$ & 14.39 (3.36) & 0.07 * \\
\hline Social & $15.20(3.01)$ & $15.07(3.11)$ & $15.72(2.54)$ & $14.48(3.53)$ & 0.08 \\
\hline Environment & $14.32(2.40)$ & $14.31(2.45)$ & $14.51(2.22)$ & $13.86(2.50)$ & 0.40 \\
\hline Spiritual & $14.66(3.65)$ & $14.34(3.70)$ & $15.43(3.27)$ & $14.69(4.18)$ & 0.04 * \\
\hline
\end{tabular}

HAART: highly active antiretroviral therapy; IIN: integrase inhibitor; MSM: men who have sex with men; NNRTI: non-nucleoside reverse transcriptase inhibitor; NRTI: nucleoside reverse transcriptase inhibitor; PI: protease inhibitor; MTR-DTG: multi-tablet regimen once daily with dolutegravir - TDF/3TC + DTG; MTR-other: multi-tablet multi-dose; SD: standard deviation; STR: single tablet regimen - TDF/3TC/EFV.

* Statistical difference. 


\section{Table 2}

Individual answers to the eight items Morisky Therapeutic Adhesion Scale (MMAS-8) and summary scores, according to the antiretroviral regimen used. Belo Horizonte, Minas Gerais State, Brazil, 2017 ( $n=393)$.

\begin{tabular}{|c|c|c|c|c|}
\hline MMAS-8 items * & $\begin{array}{c}\text { Global }(n=393) \\
n(\%)\end{array}$ & $\begin{array}{c}\text { STR }(n=254) \\
n(\%)\end{array}$ & $\begin{array}{c}\text { MTR-DTG }(n=106) \\
n(\%)\end{array}$ & $\begin{array}{c}\text { MTR-other }(n=33) \\
n(\%)\end{array}$ \\
\hline 1. Do you sometimes forget to take your medicine? (no) & $311(79.1)$ & $202(79.5)$ & $84(79.2)$ & $25(75.8)$ \\
\hline $\begin{array}{l}\text { 2. Thinking over the past } 2 \text { weeks, were there any days } \\
\text { when you did not take your medicine? (no) }\end{array}$ & $328(83.5)$ & $209(82.3)$ & $95(89.6)$ & $24(72.7)$ \\
\hline $\begin{array}{l}\text { 3. Have you ever cut back or stopped taking your } \\
\text { medicine without telling your doctor because you felt } \\
\text { worse when you took it? (no) }\end{array}$ & $375(95.4)$ & $240(94.5)$ & $106(100.0)$ & $29(87.9)$ \\
\hline $\begin{array}{l}\text { 4. When you travel or leave home, do you sometimes } \\
\text { forget to bring along your medicine? (no) }\end{array}$ & $361(91.9)$ & $229(90.2)$ & $100(94.3)$ & $32(97.0)$ \\
\hline 5. Did you take all your medicines yesterday? (yes) & $355(90.3)$ & $225(88.6)$ & $103(97.2)$ & $27(81.8)$ \\
\hline $\begin{array}{l}\text { 6. When you feel like your symptoms are under control, } \\
\text { do you sometimes stop taking your medicine? (no) }\end{array}$ & $384(97.7)$ & $247(97.2)$ & $106(100.0)$ & $31(93.9)$ \\
\hline $\begin{array}{l}\text { 7. Do you ever feel hassled about sticking to your } \\
\text { treatment plan? (no) }\end{array}$ & $317(80.7)$ & $202(79.5)$ & $92(86.8)$ & $23(69.7)$ \\
\hline $\begin{array}{l}\text { 8. How often do you have difficulty remembering to take } \\
\text { all your medicine? (never) }\end{array}$ & $258(65.6)$ & $170(66.9)$ & $64(60.4)$ & $24(72.7)$ \\
\hline \multirow[t]{2}{*}{ Adherence prevalence $[95 \% \mathrm{Cl}]$} & $176(44.8)$ & $110(43.3)$ & $53(50.0)$ & $13(39.4)$ \\
\hline & {$[39.4 ; 50.1]$} & {$[36.9 ; 49.4]$} & {$[40.4 ; 59.6]$} & {$[22.2 ; 57.9]$} \\
\hline
\end{tabular}

95\%Cl: 95\% confidence interval; MTR-DTG: multi-tablet regimen once daily with dolutegravir - TDF/3TC + DTG; MTR-other: multi-tablet multi-dose; STR: single tablet regimen - TDF/3TC/EFV.

* Use of the MMAS is protected by U.S. Copyright Laws. Permission for use is required. A license agreement is available from MMAS Research LLC 14725 NE 20th St. Bellevue WA 98007 or from dmorisky@gmail.com.

Additionally, the estimates of the prevalence of adherence to HAART according to the characteristics of the populations within the STR, MTR-DTG and MTR-other subgroups followed the same trend of the overall population, with few exceptions (Table 3).

\section{Multivariate analysis}

Figure 1 shows the results of the multivariate modeling conducted to compare the chance of adherence according to the potential explanatory factors. For the overall sample, the use of HAART regimens containing MTR-DTG ( $\mathrm{p}=0.01$ ), receiving and understanding counseling about HAART $(\mathrm{p}=0.03)$ and the physical and social domains of quality of life $(\mathrm{p}<0.04)$ were associated with higher chance of HAART adherence, while the use of illicit drugs at the lifetime $(\mathrm{p}<0.01)$ was associated with a lower chance. In addition, the presence of AIDS-defining symptoms showed a trend to be associated with higher adherence ( $\mathrm{p}=0.05)$ (Figure 1a).

Multivariate analysis restricted to the STR subgroup showed self-perception of treatment as easy ( $p=0.04)$, receiving and understanding counseling about HAART $(p=0.04)$, and the social domain of quality of life $(\mathrm{p}=0.04)$ were associated with higher chance of adherence, while having used illicit drugs at lifetime $(\mathrm{p}=0.01)$ and occurrence of adverse reactions $(\mathrm{p}=0.07)$ were associated with lower chance, although the adverse reactions results were not statistically significant (Figure 1b). For the MTR-DTG and MTR-other subgroups, quality of life domains were associated with higher chance of HAART adherence: the physical domain $(\mathrm{p}=0.01)$ for individuals in the MTR-DTG group and the psychological domain ( $\mathrm{p}=0.03$ ) for individuals in the MTR-other group (Figure 1c and 1d). In addition, being employed (0.07) and the presence of AIDS-defining symptoms ( $\mathrm{p}=0.09)$ showed a trend to be associated with a higher adherence chance among the MTR-DTG group (Figure 1c). 
Table 3

Prevalence of adherence to highly active antiretroviral therapy (HAART), according to the antiretroviral regimen used and other characteristics of individuals living with HIV. Belo Horizonte, Minas Gerais State, Brazil, $2017(\mathrm{n}=393)$.

\begin{tabular}{|c|c|c|c|c|c|c|c|c|}
\hline \multirow[t]{2}{*}{ Characteristics } & \multicolumn{2}{|c|}{$\begin{array}{l}\text { Global } \\
(n=393)\end{array}$} & \multicolumn{2}{|c|}{$\begin{array}{c}\text { STR } \\
(n=254)\end{array}$} & \multicolumn{2}{|c|}{$\begin{array}{l}\text { MTR-DTG } \\
(n=106)\end{array}$} & \multicolumn{2}{|c|}{$\begin{array}{l}\text { MTR-other } \\
\quad(n=33)\end{array}$} \\
\hline & n (\%) & $95 \% \mathrm{Cl}$ & n (\%) & $95 \% \mathrm{Cl}$ & n (\%) & $95 \% \mathrm{Cl}$ & n (\%) & $95 \% \mathrm{Cl}$ \\
\hline \multicolumn{9}{|l|}{ Sociodemographic } \\
\hline \multicolumn{9}{|l|}{ Sex } \\
\hline Male & $145(44.9)$ & $39.4 ; 50.5$ & $93(44.5)$ & $37.6 ; 51.5$ & $43(47.8)$ & $37.1 ; 58.6$ & $9(37.5)$ & $18.8 ; 59.4$ \\
\hline Female & $30(43.5)$ & $31.6 ; 56.0$ & $17(37.8)$ & $23.8 ; 53.5$ & $9(60.0)$ & $32.3 ; 83.7$ & $4(44.4)$ & $13.7 ; 78.8$ \\
\hline \multicolumn{9}{|l|}{ Age (years) } \\
\hline$\leq 33$ & $78(38.8)$ & $32.0 ; 45.9$ & $52(38.0)$ & $29.8 ; 46.6$ & $25(44.6)$ & $31.3 ; 58.5$ & $1(12.5)$ & $0.3 ; 52.7$ \\
\hline$>33$ & $98(51.0)$ & $43.7 ; 58.3$ & $58(49.6)$ & $40.2 ; 59.0$ & $28(56.0)$ & $41.3 ; 70.0$ & $12(48.0)$ & $27.8 ; 68.7$ \\
\hline \multicolumn{9}{|c|}{ Schooling level (years) } \\
\hline$\leq 9$ & $40(42.1)$ & $32.0 ; 52.7$ & $24(39.3)$ & $27.1 ; 52.7$ & $9(47.4)$ & $24.4 ; 71.1$ & $7(46.7)$ & $21.3 ; 73.4$ \\
\hline $10-12$ & $66(43.7)$ & $35.7 ; 52.0$ & $45(43.3)$ & $33.6 ; 53.3$ & $19(51.4)$ & $34.4 ; 68.1$ & $2(20.0)$ & $2.5 ; 55.6$ \\
\hline $13+$ & $70(47.6)$ & $39.3 ; 56.0$ & $41(46.1)$ & $35.4 ; 57.0$ & $25(50.0)$ & $35.5 ; 64.5$ & $4(50.0)$ & $15.7 ; 84.3$ \\
\hline \multicolumn{9}{|c|}{ Own income in the last } \\
\hline \multicolumn{9}{|l|}{6 months } \\
\hline Yes & $146(45.1)$ & $39.6 ; 50.7$ & $92(43.6)$ & $36.8 ; 50.6$ & $44(50.0)$ & $39.1 ; 60.9$ & $10(40.0)$ & $21.1 ; 61.3$ \\
\hline No & $29(42.6)$ & $30.7 ; 55.2$ & $17(40.5)$ & $25.6 ; 56.7$ & $9(50.0)$ & $26.0 ; 74.0$ & $3(37.5)$ & $8.5 ; 75.5$ \\
\hline \multicolumn{9}{|l|}{ Employment status } \\
\hline Working & $115(47.7)$ & $41.3 ; 54.2$ & $72(43.6)$ & $35.9 ; 51.6$ & $36(58.1)$ & $44.8 ; 70.5$ & $7(50.0)$ & $23.0 ; 77.0$ \\
\hline Not working & $61(40.1)$ & $32.3 ; 48.4$ & $38(42.7)$ & $32.3 ; 53.6$ & $17(38.6)$ & $24.4 ; 54.5$ & $6(31.6)$ & $12.6 ; 56.6$ \\
\hline \multicolumn{9}{|l|}{ Economic class } \\
\hline A-B & $64(45.7)$ & $37.3 ; 54.3$ & $42(44.2)$ & $34.0 ; 54.8$ & $20(52.6)$ & $35.8 ; 69.0$ & $2(28.6)$ & $3.7 ; 71.0$ \\
\hline C & $88(48.6)$ & $41.1 ; 56.1$ & $51(46.8)$ & $37.2 ; 56.6$ & $29(50.0)$ & $36.6 ; 63.4$ & $8(57.1)$ & $28.9 ; 82.3$ \\
\hline D-E & $21(33.3)$ & $22.0 ; 46.3$ & $14(32.6)$ & $19.1 ; 48.5$ & $4(40.0)$ & $12.2 ; 73.8$ & $3(30.0)$ & $6.7 ; 65.2$ \\
\hline \multicolumn{9}{|l|}{ Marital status } \\
\hline $\begin{array}{l}\text { Single/Divorced/ } \\
\text { Widowed }\end{array}$ & $134(42.8)$ & $37.3 ; 48.5$ & $84(41.4)$ & $34.5 ; 48.5$ & $41(46.1)$ & $35.4 ; 57.0$ & $9(42.9)$ & $21.8 ; 66.0$ \\
\hline $\begin{array}{l}\text { Married/Stable } \\
\text { union }\end{array}$ & $42(52.5)$ & $41.0 ; 63.8$ & $26(51.0)$ & $36.6 ; 65.2$ & $12(70.6)$ & $44.0 ; 89.7$ & $4(33.3)$ & $9.9 ; 65.1$ \\
\hline \multicolumn{2}{|l|}{ habits of life } & & & & & & & \\
\hline \multicolumn{9}{|l|}{ Alcohol use } \\
\hline Yes & $137(43.8)$ & $38.2 ; 49.5$ & $89(43.0)$ & $36.2 ; 50.0$ & $40(48.8)$ & $37.6 ; 60.1$ & $8(33.3)$ & $15.6 ; 55.3$ \\
\hline No & $39(48.8)$ & $37.4 ; 60.2$ & $21(44.7)$ & $30.2 ; 59.9$ & $13(54.2)$ & $32.8 ; 74.4$ & $5(55.6)$ & $21.2 ; 86.3$ \\
\hline \multicolumn{9}{|l|}{ Currently smoker } \\
\hline Yes & $42(41.2)$ & $31.5 ; 51.4$ & 25 (37.9) & $26.2 ; 50.7$ & $15(51.7)$ & $32.5 ; 70.6$ & $2(28.6)$ & $3.7 ; 71.0$ \\
\hline No & $134(46.0)$ & $40.2 ; 52.0$ & $85(45.2)$ & $38.0 ; 52.6$ & $38(49.4)$ & $37.8 ; 61.0$ & $11(42.3)$ & $23.4 ; 63.1$ \\
\hline \multicolumn{9}{|l|}{$\begin{array}{l}\text { Ever used any illicit } \\
\text { drug }\end{array}$} \\
\hline Yes & $73(37.4)$ & $30.6 ; 44.6$ & $42(34.1)$ & $25.8 ; 43.2$ & $27(45.0)$ & $32.1 ; 58.4$ & $4(33.3)$ & $9.9 ; 65.1$ \\
\hline No & $103(52.3)$ & $45.1 ; 59.4$ & $68(51.9)$ & $43.0 ; 60.7$ & $26(56.5)$ & $41.1 ; 71.1$ & $9(45.0)$ & $23.1 ; 68.5$ \\
\hline \multicolumn{9}{|l|}{ Condom use } \\
\hline Yes & $95(42.8)$ & $36.2 ; 49.6$ & $56(39.7)$ & $31.6 ; 48.3$ & $32(50.0)$ & $37.2 ; 62.8$ & $7(41.2)$ & $18.4 ; 67.1$ \\
\hline No & $43(45.7)$ & $35.4 ; 56.3$ & $31(44.9)$ & $32.9 ; 57.4$ & $11(55.0)$ & $31.5 ; 76.9$ & $1(20.0)$ & $0.5 ; 71.6$ \\
\hline \multicolumn{9}{|c|}{ Risk/Exposure category } \\
\hline MSM & $90(45.5)$ & $38.4 ; 52.7$ & $59(44.0)$ & $35.5 ; 52.9$ & $25(48.1)$ & $34.0 ; 62.4$ & $6(50.0)$ & $21.1 ; 78.9$ \\
\hline Other & 81 (44.5) & $37.2 ; 52.0$ & $47(41.6)$ & $32.4 ; 51.2$ & $27(55.1)$ & $40.2 ; 69.3$ & $7(35.0)$ & $15.4 ; 59.2$ \\
\hline
\end{tabular}

(continues) 
Table 3 (continued)

\begin{tabular}{|c|c|c|c|c|c|c|c|c|}
\hline \multirow[t]{2}{*}{ Characteristics } & \multicolumn{2}{|c|}{$\begin{array}{l}\text { Global } \\
(n=393)\end{array}$} & \multicolumn{2}{|c|}{$\begin{array}{c}\text { STR } \\
(n=254)\end{array}$} & \multicolumn{2}{|c|}{$\begin{array}{c}\text { MTR-DTG } \\
(n=106)\end{array}$} & \multicolumn{2}{|c|}{$\begin{array}{l}\text { MTR-other } \\
\quad(n=33)\end{array}$} \\
\hline & n (\%) & $95 \% \mathrm{Cl}$ & n (\%) & $95 \% \mathrm{Cl}$ & n (\%) & $95 \% \mathrm{Cl}$ & n (\%) & $95 \% \mathrm{Cl}$ \\
\hline \multicolumn{9}{|l|}{ Clinical } \\
\hline \multicolumn{9}{|l|}{ AIDS defining illness } \\
\hline Non-AIDS & $131(43.1)$ & $37.5 ; 48.9$ & $78(41.1)$ & $34.0 ; 48.4$ & $47(49.5)$ & $39.1 ; 59.9$ & $6(31.6)$ & $12.6 ; 56.6$ \\
\hline AIDS & $40(52.6)$ & $40.8 ; 64.2$ & $28(49.1)$ & $35.6 ; 62.7$ & $5(83.3)$ & $35.9 ; 99.6$ & $7(53.8)$ & $25.1 ; 80.8$ \\
\hline \multicolumn{9}{|l|}{ Anxiety } \\
\hline Yes & $48(39.0)$ & $30.4 ; 48.2$ & $31(38.3)$ & $27.7 ; 49.7$ & $14(50.0)$ & $30.6 ; 69.4$ & $3(21.4)$ & $4.7 ; 50.8$ \\
\hline No & $128(47.4)$ & $41.3 ; 53.5$ & $79(45.7)$ & $38.1 ; 53.4$ & $39(50.0)$ & $38.5 ; 61.5$ & $10(52.6)$ & $28.9 ; 75.6$ \\
\hline \multicolumn{9}{|l|}{ Depression } \\
\hline Yes & $24(28.6)$ & $19.2 ; 39.5$ & $17(28.8)$ & $17.8 ; 42.1$ & $5(33.3)$ & $11.8 ; 61.6$ & $2(20.0)$ & $2.5 ; 55.6$ \\
\hline No & $152(49.2)$ & $43.5 ; 54.9$ & $93(47.7)$ & $40.5 ; 54.9$ & $48(52.7)$ & $42.0 ; 63.3$ & $11(47.8)$ & $26.8 ; 69.4$ \\
\hline \multicolumn{9}{|l|}{ Comorbidities } \\
\hline Yes & 35 (44.9) & $33.6 ; 56.6$ & $24(47.1)$ & $32.9 ; 61.5$ & $8(61.5)$ & $31.6 ; 86.1$ & $3(21.4)$ & $4.7 ; 50.8$ \\
\hline No & $141(44.8)$ & $39.2 ; 50.4$ & $86(42.4)$ & $35.5 ; 49.5$ & $45(48.4)$ & $37.9 ; 59.0$ & $10(52.6)$ & $28.9 ; 75.6$ \\
\hline \multicolumn{9}{|l|}{ Coinfections } \\
\hline Yes & $13(46.4)$ & $27.5 ; 66.1$ & $11(50.0)$ & $28.2 ; 71.8$ & $2(100.0)$ & $100.0 ; 100.0$ & $0(0.0)$ & $0.0 ; 60.2$ \\
\hline No & $162(44.6)$ & $39.5 ; 49.9$ & $98(42.6)$ & $36.1 ; 49.3$ & $51(49.0)$ & $39.1 ; 59.0$ & $13(44.8)$ & $26.4 ; 64.3$ \\
\hline \multicolumn{9}{|l|}{ HAART-related } \\
\hline \multicolumn{9}{|l|}{$\begin{array}{l}\text { Classical therapeutic } \\
\text { regimen }\end{array}$} \\
\hline $2 \mathrm{NRTI}+1 \mathrm{NNRTI}$ & $115(43.9)$ & $37.8 ; 50.1$ & $110(43.3)$ & $37.1 ; 49.6$ & * & * & $5(62.5)$ & $24.5 ; 91.5$ \\
\hline $2 \mathrm{NRTI}+1 \mathrm{IIN}$ & $54(49.1)$ & $39.4 ; 58.8$ & - & - & $53(50.0)$ & $40.1 ; 59.9$ & $1(25.0)$ & $0.6 ; 80.6$ \\
\hline $2 \mathrm{NRTI}+2 \mathrm{PI}$ & $7(33.3)$ & $14.6 ; 57.0$ & - & - & * & * & $7(33.3)$ & $14.6 ; 57.0$ \\
\hline \multicolumn{9}{|l|}{ Adverse reactions } \\
\hline Yes & $141(42.1)$ & $36.8 ; 47.6$ & $92(40.7)$ & $34.2 ; 47.4$ & $40(49.4)$ & $38.1 ; 60.7$ & $9(32.1)$ & $15.9 ; 52.4$ \\
\hline No & $34(61.8)$ & $47.7 ; 74.6$ & $17(65.4)$ & $44.3 ; 82.8$ & $13(52.0)$ & $31.3 ; 72.2$ & $4(100.0)$ & $100.0 ; 100.0$ \\
\hline \multicolumn{9}{|l|}{$\begin{array}{l}\text { Number of adverse } \\
\text { reactions }\end{array}$} \\
\hline$\leq 5$ & $140(49.5)$ & $43.5 ; 55.5$ & $81(48.5)$ & $40.7 ; 56.3$ & $49(51.0)$ & $40.6 ; 61.4$ & $10(50.0)$ & $27.2 ; 72.8$ \\
\hline$>5$ & 35 (32.7) & $24.0 ; 42.5$ & $28(32.9)$ & $23.1 ; 44.0$ & $4(40.0)$ & $12.2 ; 73.8$ & $3(25.0)$ & $5.5 ; 57.2$ \\
\hline \multicolumn{9}{|l|}{$\begin{array}{l}\text { Self-perception of } \\
\text { treatment difficulty }\end{array}$} \\
\hline Easy & $139(50.5)$ & $44.5 ; 56.6$ & $87(50.3)$ & $42.6 ; 58.0$ & $44(50.6)$ & $39.6 ; 61.5$ & $8(53.3)$ & $26.6 ; 78.7$ \\
\hline Difíccult & 37 (31.9) & $23.6 ; 41.2$ & $23(29.1)$ & $19.4 ; 40.4$ & $9(47.4)$ & $24.4 ; 71.1$ & $5(27.8)$ & $9.7 ; 53.5$ \\
\hline \multicolumn{9}{|c|}{ Time on HAART (days) } \\
\hline$\leq 60$ & $87(49.2)$ & $41.6 ; 56.8$ & $46(45.5)$ & $35.6 ; 55.8$ & $37(54.4)$ & $41.9 ; 66.5$ & $4(50.0)$ & $15.7 ; 84.3$ \\
\hline$>60$ & 89 (41.6) & $34.9 ; 48.5$ & $64(42.1)$ & $34.2 ; 50.4$ & $16(42.1)$ & $26.3 ; 59.2$ & $9(37.5)$ & $18.8 ; 59.4$ \\
\hline \multicolumn{9}{|l|}{$\begin{array}{l}\text { Laboratorial } \\
\text { (before HAART) }\end{array}$} \\
\hline \multicolumn{9}{|l|}{$\begin{array}{l}\text { CD4+ T lymphocyte } \\
\text { count (cells/mm3) }\end{array}$} \\
\hline$\leq 200$ & $44(50.0)$ & $39.1 ; 60.9$ & $34(54.0)$ & $40.9 ; 66.6$ & $7(50.0)$ & $23.0 ; 77.0$ & $3(27.3)$ & $6.0 ; 61.0$ \\
\hline$>200$ & $99(41.6)$ & $35.3 ; 48.1$ & $58(39.5)$ & $31.5 ; 47.8$ & $36(47.4)$ & $35.8 ; 59.2$ & $5(33.3)$ & $11.8 ; 61.6$ \\
\hline \multicolumn{9}{|l|}{ Viral load (copies/mL) } \\
\hline$\leq 100,000$ & $105(43.6)$ & $37.2 ; 50.1$ & $67(43.8)$ & $35.8 ; 52.0$ & $33(45.8)$ & $34.0 ; 58.0$ & $5(31.3)$ & $11.0 ; 58.7$ \\
\hline$>100,000$ & $40(46.0)$ & $35.2 ; 57.0$ & $27(45.0)$ & $32.1 ; 58.4$ & $8(53.3)$ & $26.6 ; 78.7$ & $5(41.7)$ & $15.2 ; 72.3$ \\
\hline
\end{tabular}

(continues) 
Table 3 (continued)

\begin{tabular}{|c|c|c|c|c|c|c|c|c|}
\hline \multirow[t]{2}{*}{ Characteristics } & \multicolumn{2}{|c|}{$\begin{array}{c}\text { Global } \\
(n=393)\end{array}$} & \multicolumn{2}{|c|}{$\begin{array}{c}\text { STR } \\
(n=254)\end{array}$} & \multicolumn{2}{|c|}{$\begin{array}{c}\text { MTR-DTG } \\
(n=106)\end{array}$} & \multicolumn{2}{|c|}{$\begin{array}{c}\text { MTR-other } \\
(n=33)\end{array}$} \\
\hline & n (\%) & $95 \% \mathrm{Cl}$ & n (\%) & $95 \% \mathrm{Cl}$ & n (\%) & $95 \% \mathrm{Cl}$ & n (\%) & $95 \% \mathrm{Cl}$ \\
\hline \multicolumn{9}{|l|}{$\begin{array}{l}\text { Health service- } \\
\text { related }\end{array}$} \\
\hline \multicolumn{9}{|l|}{ Healthcare facility } \\
\hline । & $68(46.9)$ & $38.6 ; 55.4$ & $59(47.2)$ & $38.2 ; 56.3$ & $0(0.0)$ & * & $9(45.0)$ & $23.1 ; 68.5$ \\
\hline$\|$ & $71(43.3)$ & $35.6 ; 51.2$ & $43(40.6)$ & $31.1 ; 50.5$ & $25(53.2)$ & $38.1 ; 67.9$ & $3(27.3)$ & $6.0 ; 61.0$ \\
\hline III & $37(44.0)$ & $33.2 ; 55.3$ & $8(34.8)$ & $16.4 ; 57.3$ & $28(47.5)$ & $34.3 ; 60.9$ & $1(50.0)$ & $1.3 ; 98.7$ \\
\hline \multicolumn{9}{|c|}{$\begin{array}{l}\text { Receiving and } \\
\text { understanding HAART } \\
\text { counseling }\end{array}$} \\
\hline Yes & $160(46.6)$ & $41.3 ; 52.1$ & $103(46.4)$ & $39.7 ; 53.2$ & $45(48.4)$ & $37.9 ; 59.0$ & $12(42.9)$ & $24.5 ; 62.8$ \\
\hline No & 15 (31.3) & $18.7 ; 46.3$ & $6(20.0)$ & $7.7 ; 38.6$ & $8(61.5)$ & $31.6 ; 86.1$ & $1(20.0)$ & $0.5 ; 71.6$ \\
\hline
\end{tabular}

95\%Cl: 95\% confidence interval; HAART: highly active antiretroviral therapy; IIN: integrase inhibitor; MSM: men who have sex with men; NNRTI: nonnucleoside reverse transcriptase inhibitor; NRTI: nucleoside reverse transcriptase inhibitor; PI: protease inhibitor; MTR-DTG: multi-tablet regimen once daily with dolutegravir - TDF/3TC + DTG; MTR-other: multi-tablet multi-dose; STR: single tablet regimen - TDF/3TC/EFV.

* Not estimable.

All models presented a good fit, with Hosmer-Lemeshow test and the ROC curve values lower than 0.05 and higher than 0.7 , respectively. Specific values were $\mathrm{p}=0.17$ and $\mathrm{ROC}=0.7$ for the global model, $\mathrm{p}=0.55$ and ROC $=0.7$ for STR model, $\mathrm{p}=0.48$ and ROC $=0.7$ for MTR-DTG model and $\mathrm{p}=0.41$ and $\mathrm{ROC}=0.8$ for the MTR-other model.

\section{Discussion}

In this cross-sectional study, which included individuals at the beginning of treatment, the overall rate of adherence was $44.8 \%$, measured by the MMAS- 8 self-report scale. Despite having no differences between the HAART subgroups in the raw analysis, multivariate modeling showed a higher chance of adherence among patients using the multi-table once-daily regimen containing TDF/3TC + DTG. In addition, different factors influenced the adherence in each subgroup.

Regarding the characteristics of the sample, there was a predominance of males, MSM, aged less than 33 years, with 10 to 12 years of schooling, with their own income in the last six months, employed and single, divorced or widowed. These results are consistent with the profile of the Brazilian people living with HIV and the trend of increase of the HIV epidemic among young MSM 6,20.

The overall prevalence of adherence observed in the present study was similar to the ones reported in Brazil in earlier years 21,22 and worldwide 23,24,25. Previous studies using the MMAS-8 with the same cut-off point as ours reported a prevalence of adherence of $43.7 \% 24$ and $61.7 \% 25$, while studies using other self-report measurements of adherence reported rates above $70 \% 3,26,27$.

The rate of adherent individuals within the HAART subgroups was lower in the present study when compared to the literature. Studies using self-report scales showed rates of adhesion ranging from $62 \%$ to $85.4 \%$ for the STR group and from $48.6 \%$ to $92.8 \%$ for MTR groups 26,27 . However, when a higher cut-off point in the MMAS- 8 is used (100\%), reported rates can be as lower as $14.2 \%$ for STR and $26.9 \%$ for MTR 23 .

Studies evaluating adherence specifically in MTR-DTG regimens are scarce, and those evaluating HAART regimens of multi-tablet once-daily also reported higher rates of adherent individuals than our study: $65.3 \% 28$ and $68.9 \%$ 7. However, they measured adherence using pharmacy refill records and tablet counts, respectively.

There are plenty of methods to measure adherence and the differences between them impact on the results and its comparability across studies. Indirect methods, like self-report, can be measured 
Figure 1

Forest plot of multivariate analysis of adherence to highly active antiretroviral therapy (HAART) according to the antiretroviral regimen.

1a) Global analysis $(n=393)$

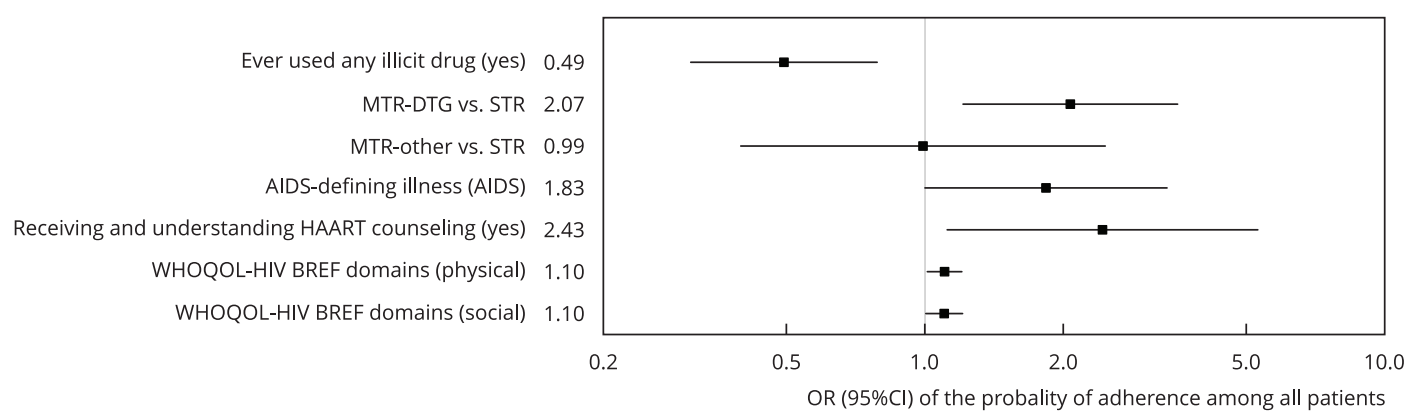

1b) $\operatorname{STR}(n=254)$

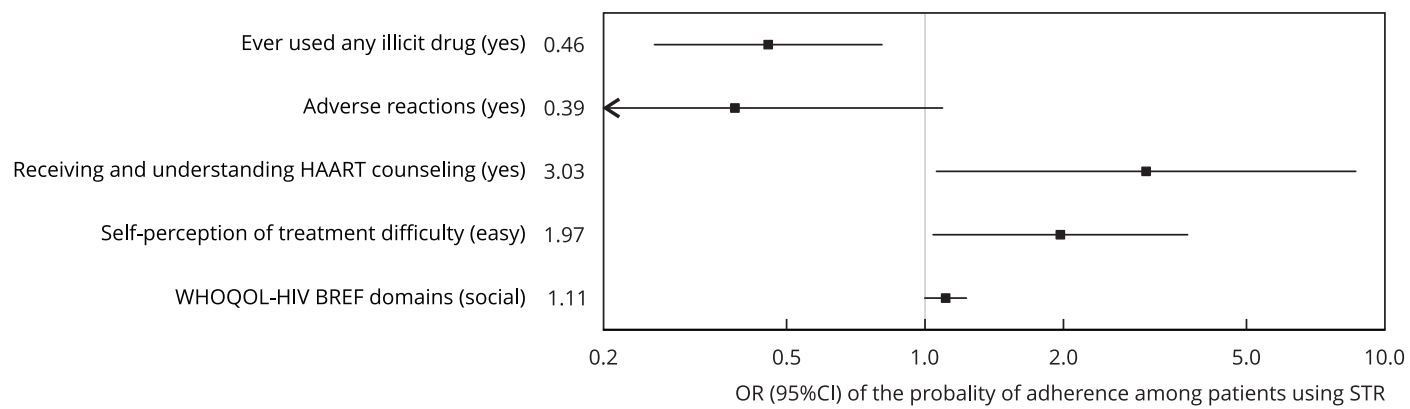

(continues)

by direct questions to patients or using scales, such as the MMAS-8, the Visual Analogue Scale and the Questionnaire for the Evaluation of Adherence to Antiretroviral Treatment. Another issue is related to the cut-off points adopted to define adherence. Most studies use a cut-off point up to $95 \%$, while in the present study a more rigorous cut-off point was adopted (100\%). Additionally, the specificity of the Brazilian therapeutic regimens, as well as the cultural and sociodemographic characteristics of the population, can explain the variability in the results.

The overall adherence to antiretroviral therapy was influenced by behavioral and lifestyle habits, clinical, HAART and health service-related characteristics and by the quality of life. Garbin et al. 29 found in a literature review a rate of adherence to antiretroviral therapy ranged from $18 \%$ to $74.3 \%$ among Brazilian states.

There are several studies in the literature reporting better adherence rates among patients using STR when compared to multi-dose MTR 7,8,28,30. The meta-analysis of Nachega et al. 8 showed better adherence for HAART-naïve patients using once-daily regimens, which included STR and other regimens taken once-daily in the same group, compared to twice-daily regimens. Results for the comparison of STR to once-daily MTR, however, are more heterogeneous, with studies reporting better results to STR or absence of difference among groups 7,28.

Contrastingly, in our study, the MTR once-daily containing DTG showed better results when compared to the STR. Both regimens are used with the "backbone" of TDF/3TC, and bottom line, the comparison of effects are related to the third antiretroviral agent characteristics, such as their pill burden and tolerability. We observed a lower rate of adverse events and a lower proportion of patients 
1c) MTR-DTG $(n=106)$

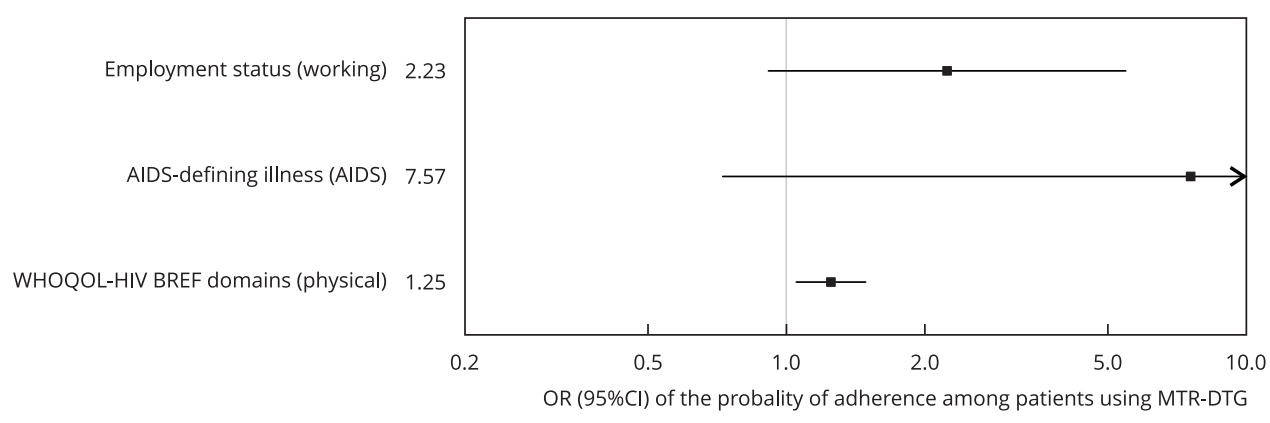

1d) MTR-other $(n=33)$

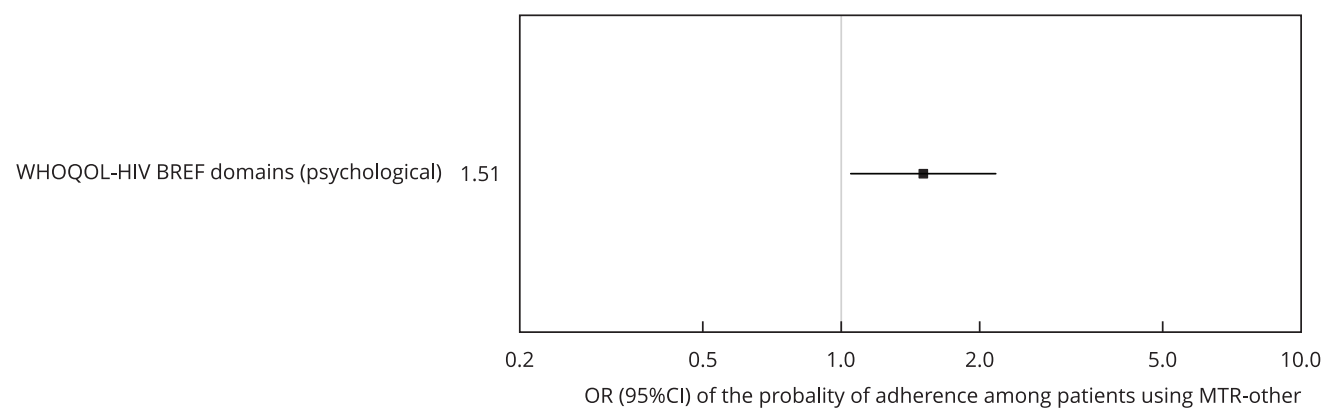

95\%Cl: 95\% confidence interval; MTR-DTG: multi-tablet regimen once daily with dolutegravir - TDF/3TC + DTG; MTR-other: multi-tablet multi-dose; OR: odds ratio; STR: single tablet regimen - TDF/3TC/EFV.

having more than five adverse drug reactions among patients in the MTR-DTG group. The profile of adverse events also differenced among groups 31 and may have contributed to increasing the chance of adherence to HAART. Adverse reactions such as dizziness, rashes, and vomiting are common and may interfere negatively with the patient's daily life. Discussing all possible adverse reactions before starting HAART and managing the symptoms are critical for achieving a good adherence. Additionally, individual evaluation of adverse reactions can be useful in the elaboration of strategies to maintain a good adherence 32 .

The association between quality of life and adherence is common in the literature, and studies have reported a higher quality of life and its components, such as the capacity of working, social support and psychological status to influence the success of HAART 33 . In our study, patients on MTR-other regimens showed, in general, a worse prognosis compared to other groups, illustrated by the higher rate of advanced disease, a higher number of comorbidities and higher adverse events rate. In addition to lower adherence rates, all these characteristics may affect their social role and self-perception on their health status, reflecting the lower quality of life values among this group.

The quality of the counseling to the patient about their HAART treatment and the good relationship between patient and health professionals also influenced positively the adherence 24,34 . The counseling about medicines help the individual to better understand his/her treatment and this contributes for patients to strive more to follow it correctly. Indeed, individual's perception of their treatment as easy was associated with higher chance of adherence to HAART in the STR subgroup. Previous studies have shown individuals who considered their treatment difficult were 21 times more likely to have inadequate adherence 35 . 
Another concern is with the use of illicit drugs, as they affected negatively the adherence to HAART in this and in several other studies 27,32 . Some possible strategies to increase adherence include treating the addiction, preparing health professionals to deal with these individuals, and seeking healthier alternatives for coping together with patients 36,37 . Therefore, investing in strategies to improve and broaden the counseling by health professionals is of great importance, since this type of strategy can target simultaneously various factors related to adherence, has low cost, easy implementation and results are achieved in the short to medium term. In addition, health professionals should individually approach patient's self-perception in order to clarify possible doubts and improve their own perception 35 .

Low adherence to HAART is a public health issue, as it influences not only the health of each individual but also the overall population living with HIV. In addition to being a major cause of therapeutic failure, it increases the risk of developing drug resistance. The transmission of resistant strains to the initial therapeutic regimens contributes to the bankruptcy of these regimens, leading to negative impacts on public policies and on the health system 38. Clinically, one of the outcomes of inadequate adherence is the reduction of LT-CD4+ leading to a decreased immunity level, increased risk of opportunistic infections, and possible progression to AIDS. These factors may also result in an increase in the risk of hospitalizations and consequently higher costs to the health system 38,39.

Strategies to achieve adequate HAART adherence include cognitive-behavioral therapy, performed in motivational interviews and followed by educational actions, choice of supporters and medicines reminder devices, such as cell phone alarms and tablet packaging with timers or alarms 40 . Short message service, such as SMS text messaging, also increase adherence compared to standard care and combined interventions resulted in greater overall adherence than isolated interventions 41 .

Despite the adherence results, a study with participants of service I showed, at six months, 74.6\% of overall viral suppression and $80.6 \%$ of viral supression among patients who used STR ( $\mathrm{p}=0.04)$. Factors independently associated with viral suppression at six months were: (negatively) viral load $\geq 100,000$ copies/mL, symptoms of AIDS, longer interval time between diagnosis and initiation of antiretroviral therapy, antiretroviral switching, smoking or current illicit drugs usage; and (positively) category of exposure of men who have sex with men and acherence to HAART 42.

An international cohort study in South Carolina (USA) showed that people using STR achieve viral suppression with adherence levels of $80 \%$ or greater, whereas people using MTR require adherence levels of $90 \%$ or greater to achieve viral suppression 43.

In Brazil, the Ministry of Health recommends the support of the multi-professional team to the individual, including the implementation of support groups, lectures, activities in waiting rooms, educational activities with the availability of material and actions in partnership with civil society organizations. Furthermore, it is important health professionals to well-inform individuals in a clear way, making sure they understand the information. In addition, it is important to understand patients' beliefs and routines to determine together the best way to achieve adherence 4 .

High-quality HIV services must continue to expand in order to maintain and improve the outcomes. Brazil has seen an increase from 83\% (in 2015) to 84\% (in 2017) in HIV-diagnosis. The portion of people living with HIV on treatment stood at $64 \%$ in $2017,59 \%$ of whom were virally suppressed. One major challenge is the sustained programs in the country for reducing the number of new infections among key affected populations 4.

This study has some limitations. The adherence was measured by a self-report scale not specific to HIV. However, this method is fast, easy to apply and is a good predictor of virological failure 44 . Furthermore, several studies used the MMAS- 8 to measure HAART adherence 23,24,25,35 and although there is a multiplicity of indirect measures of adherence, none of them is considered a "gold standard”. The triangulation of methods increases the validity and reliability of the adherence results. For greater robustness of results and to allow comparisons between them, future research and practice interventions should use a standardized and internationally accepted definition; clearly describing which medicines and methods were used.

In addition, due to the cross-sectional design of the study, it is not possible to establish the temporal relationship between cause and effect. However, we continue to follow up patients to evaluate long-term results and to confirm these findings. Finally, the study's population was selected by nonrandomized sampling although we have universal register on the SICLOM. 


\section{Conclusion}

The overall adherence rate in individual's initiating HAART was low with better results for patients using the dolutegravir once-daily regimen, which is the first-choice regimen currently used in Brazil. Results highlighted the need for intervention strategies to increase adherence to HAART, focusing on health professional's counseling about HAART and substance use. Finally, the interventions performed should be specific and oriented to the HAART-regimen, since different factors affect adherence in each group.

\section{Contributors}

T. S. Cardoso was responsible for study design, data extraction, data interpretation, drafting of the manuscript and approval of the final manuscript. J. O. Costa was responsible for data extraction, data analyses, data interpretation, drafting of the manuscript and critical review. E. A. Reis was responsible for data interpretation, critical review, and approval of the final manuscript. M. R. Silveira was responsible for study design, critical review, and approval of the final manuscript. P. F. Bonolo was responsible for study design, data interpretation, and critical review. S. F. Santos was responsible for data interpretation and critical review. M. G. B. Ceccato was responsible for study design, data analyses, data interpretation, critical review, and approval of the final manuscript.

\section{Additional informations}

ORCID: Tarsilla Spezialli Cardoso (0000-00028400-1689); Juliana de Oliveira Costa (0000-00028355-023X); Edna Afonso Reis (0000-0003-14659167); Micheline Rosa Silveira (0000-0001-70024428); Palmira De Fátima Bonolo (0000-00032744-7139); Simone Furtado Dos Santos (00000002-2060-5934); Maria das Graças Braga Ceccato (0000-0002-4340-0659).

\section{Acknowledgments}

Use of the MMAS is protected by U.S. Copyright Laws. Permission for use is required. A license agreement is available from MMAS Research LLC 14725 NE 20th St. Bellevue WA 98007 or from dmorisky@ gmail.com. To Minas Gerais State Research Foundation (FAPEMIG) for the financial support.

\section{References}

1. Nachega JB, Hislop M, Dowdy DW, Chaisson RE, Regensberg L, Maartens G. Adherence to nonnucleoside reverse transcriptase inhibitorbased HIV therapy and virologic outcomes. Ann Intern Med 2007; 146:564-73.

2. De Geest S, Sabaté E. Adherence to long-term therapies: evidence for action. Eur J Cardiovasc Nurs 2003; 2:323.

3. Airoldi M, Zaccarelli M, Bisi L, Bini T, Antinori A, Mussini C, et al. One-pill once-a-day HAART: a simplification strategy that improves adherence and quality of life of HIV-infected subjects. Patient Prefer Adherence 2010; 4:115-25.

4. Ministério da Saúde. Protocolo clínico e diretrizes terapêuticas para manejo da infecção pelo HIV em adultos. Brasília: Ministério da Saúde; 2018.

5. Melchior R, Nemes MIB, Alencar TMD, Buchalla CM. Challenges of treatment adherence by people living with HIV/AIDS in Brazil. Rev Saúde Pública 2007; 41 Suppl 2:87-93.

6. Casotti JAS, Mendes AAS, Endlich BN, Queiroz MD, Tartaglia RS, Motta TQR. Factors associated with adherence to HAART in patients with HIV/Aids. J Bras Doenças Sex Transm 2011; 23:215-21.

7. Chen Y, Chen K, Kalichman SC. Barriers to HIV medication adherence as a function of regimen simplification. Ann Behav Med 2017; 51:67-78.

8. Nachega JB, Parienti JJ, Uthman OA, Gross R, Dowdy DW, Sax PE, et al. Lower pill burden and once-daily antiretroviral treatment regimens for HIV infection: a meta-analysis of randomized controlled trials. Clin Infect Dis 2014; 58:1297-307.

9. Morisky DE, Ang A, Krousel-Wood M, Ward HJ. Predictive validity of a medication adherence measure in an outpatient setting. J Clin Hypertens 2008; 10:348-54. 
10. Morisky DE, DiMatteo MR. Improving the measurement of self-reported medication nonadherence: response to authors. J Clin Epidemiol 2011; 64:255-7.

11. Krousel-Wood M, Islam T, Webber LS, Re RN, Morisky DE, Muntner P. New medication adherence scale versus pharmacy fill rates in seniors with hypertension. Am J Manag Care 2009; 15:59-66.

12. Oliveira-Filho AD, Morisky DE, Neves SJ, Costa FA, Lyra Jr. DP. The 8-item morisky medication adherence scale: validation of a Brazilian-Portuguese version in hypertensive adults. Res Soc Adm Pharm 2014; 10:554-61.

13. Associação Brasileira de Empresas de Pesquisa. Brazil 2015 criteria and update of class distribution for 2016. São Paulo: Associação Brasileira de Empresas de Pesquisa; 2016.

14. 1993 revised classification system for HIV infection and expanded surveillance case definition for AIDS among adolescents and adults. MMWR Recomm Rep 1992; 41:1-19.

15. Zigmond AS, Snaith RP. The hospital anxiety and depression scale. Acta Psychiatr Scand 1983; 67:361-70.

16. Botega NJ, Bio MR, Zomignani MA, Garcia Jr. C, Pereira WA. Transtornos do humor em enfermaria de clínica médica e validação de escala de medida (HAD) de ansiedade e depressão. Rev Saúde Pública 1995; 29:355-63.

17. WHOQOL HIV Group. WHOQOL-HIV for quality of life assessment among people living with HIV and AIDS: results from the field test. AIDS Care 2004; 16:882-9.

18. Zimpel RR, Fleck MP. Quality of life in HIVpositive Brazilians: application and validation of the WHOQOL-HIV, Brazilian version. AIDS Care 2007; 19:923-30.

19. Landis JR, Koch GG. The measurement of observer agreement for categorical data. Biometrics 1977; 33:159-74

20. Departamento de Vigilância, Prevenção e Controle das Infecções Sexualmente Transmissíveis, do HIV/Aids e das Hepatites Virais, Secretaria de Vigilância em Saúde, Ministério da Saúde. Boletim Epidemiológico - Aids e IST 2017; Ano V, no 1.

21. Resende RC, Podestá MHMC, Souza W, Oliveira Barroso T, Costa Vilas OMG, Ferreira EB. Adesão ao tratamento antirretroviral de pacientes vivendo com HIV/AIDS atendidos pelo Sistema Único de Saúde. Revista da Universidade Vale do Rio Verde 2012; 10:186-201.

22. Nobre ACL, Matos VC. Avaliação da adesão a terapia antirretroviral de pacientes portadores de HIV. Revista Brasileira de Farmácia Hospitalar e Serviços de Saúde 2012; 3:37-41.

23. Wójcik K, Piekarska A, Jabłonowska E. Adherence to antiviral therapy in HIV or HBV-infected patients. Przegl Epidemiol 2016; 70:2732.

24. Peyre M, Gauchet A, Roustit M, Leclercq P, Epaulard O. Influence of the first consultation on adherence to antiretroviral therapy for HIV-infected patients. Open AIDS J 2016; 10:182-9.
25. Velvanathan T, Islahudin F, Sim BL, Taha NA Simplification of HAART therapy on ambulatory HIV patients in Malaysia: a randomized controlled trial. Pharm Pract (Granada) 2016; 14:830.

26. Tennant SJ, Hester EK, Caulder CR, Lu ZK, Bookstaver PB. Adherence among rural HIVinfected patients in the deep south: a comparison between single-tablet and multi-tablet once-daily regimens. J Int Assoc Provid AIDS Care 2015; 14:64-71.

27. Hanna DB, Hessol NA, Golub ET, Cocohoba JM, Cohen MH, Levine AM, et al. Increase in single-tablet regimen use and associated improvements in adherence-related outcomes in HIV-infected women. J Acquir Immune Defic Syndr 2014; 65:587-96.

28. Raffi F, Yazdanpanah Y, Fagnani F, Laurendeau C, Lafuma A, Gourmelen J. Persistence and adherence to single-tablet regimens in HIV treatment: a cohort study from the French National Healthcare Insurance Database. J Antimicrob Chemother 2015; 70:2121-8.

29. Garbin CAS, Gatto RCJ, Garbin AJI. Adesão à terapia antirretroviral em pacientes HIV soropositivos no Brasil: uma revisão da literatura. Arch Health Invest 2017; 6:65-70.

30. Yager J, Faragon J, McGuey L, Hoye-Simek A, Hecox Z, Sullivan S, et al. Relationship between single tablet antiretroviral regimen and adherence to antiretroviral and non-antiretroviral medications among veterans' affairs patients with human immunodeficiency virus. AIDS Patient Care STDS 2017; 31:370-6.

31. Mendes JC, Bonolo PF, Ceccato MDGB, Costa JO, Reis AMM, Santos H, et al. Adverse reactions associated with first-line regimens in patient initiating antiretroviral therapy. Eur J Clin Pharmacol 2018; 74:1077-88.

32. Shigdel R, Klouman E, Bhandari A, Ahmed LA. Factors associated with adherence to antiretroviral therapy in HIV-infected patients in Kathmandu District, Nepal. HIV/AIDS (Auckl) 2014; 6:109-16.

33. Geocze L, Mucci S, De Marco MA, Nogueira-Martins LA, Citero VA. Qualidade de vida e adesão ao tratamento anti-retroviral de pacientes portadores de HIV. Rev Saúde Pública 2010; 44:743-9.

34. Flickinger TE, Saha S, Moore RD, Beach MC. Higher quality communication and relationships are associated with improved patient engagement in HIV care. J Acquir Immune Defic Syndr 2013; 63:362-6.

35. Gokarn A, Narkhede MG, Pardeshi GS, Doibale MK. Adherence to antiretroviral therapy. J Assoc Physicians India 2012; 60:16-20.

36. Palepu A, Milloy MJ, Kerr T, Zhang R, Wood E. Homelessness and adherence to antiretroviral therapy among a cohort of HIV-infected injection drug users. J Urban Health 2011; 88:54555.

37. Oliveira RCS, Moraes DCA, Oliveira ECS, Cabral JRP, Pereira JFM, Santos CSS. Associação do uso de álcool e drogas e a adesão ao tratamento antirretroviral. Rev Enferm UFPE on-line 2017; 11:3862-8. 
38. Heestermans T, Browne JL, Aitken SC, Vervoort SC, Klipstein-Grobusch K. Determinants of adherence to antiretroviral therapy among HIV-positive adults in sub-Saharan Africa: a systematic review. BMJ Glob Health 2016; 1:e000125.

39. Acurcio FA, Puig-Junoy J, Bonolo PF, Ceccato MGB, Guimarães MDC. Análisis coste-efectividad de la adhesión inicial a la terapia antirretroviral entre individuos infectados por el VIH en Belo Horizonte, Brasil. Rev Esp Salud Pública 2006; 80:41-54.

40. Chaiyachati KH, Ogbuoji O, Price M, Suthar $\mathrm{AB}$, Negussie EK, Bärnighausen T. Interventions to improve adherence to antiretroviral therapy: a rapid systematic review. AIDS 2014; 28 Suppl 2:S187-204.

41. Kanters S, Park JJ, Chan K, Socias ME, Ford N, Forrest JI, et al. Interventions to improve adherence to antiretroviral therapy: a systematic review and network meta-analysis. Lancet HIV 2017; 4:e31-40.
42. Costa JO, Ceccato MGB, Silveira MR, Bonolo PF, Reis EA, Acurcio FA. Effectiveness of antiretroviral therapy in the single-tablet regimen era. Rev Saúde Pública 2018; 52:87.

43. Sutton SS, Magagnoli J, Hardin JW. Odds of viral suppression by single-tablet regimens, multiple-tablet regimens, and adherence level in HIV/AIDS patients receiving antiretroviral therapy. Pharmacotherapy 2017; 37:204-13.

44. Been SK, Yildiz E, Nieuwkerk PT, Pogány K, van de Vijver DAMC, Verbon A. Self-reported adherence and pharmacy refill adherence are both predictive for an undetectable viral load among HIV-infected migrants receiving cART. PLoS One 2017; 12:e186912. 


\section{Resumo}

Avaliamos a adesão à terapia antirretroviral (TARV) e fatores associados de acordo com o tipo de esquema em pacientes no início do tratamento em Belo Horizonte, Minas Gerais, Brasil. Mensuramos a adesão com a Escala de Adesão Terapêutica de Morisky, de oito itens (MMAS-8), e comparamos o uso de tenofovir/lamivudina com efavirenz, um comprimido uma vez ao dia (STR), ou dolutegravir em múltiplos comprimidos uma vez ao dia (MTR-DTG), com outros esquemas com múltiplos comprimidos ao dia (MTR-outros). Conduzimos uma análise de regressão logística multivariada para avaliar os fatores associados à adesão. Foram incluídos 393 pacientes: 254 em uso de STR, 106 MTR-DTG e 33 MTR-outros. A taxa global de adesão foi 44,8\% (IC95\%: 39,4; 50,1), sendo $50 \%$ para MTR-DTG, 43,3\% para STR e 39,4\% para MTR-outros. A análise multivariada mostrou chances maiores de adesão em pacientes em uso de MTR-DTG, pacientes que haviam recebido e compreendido o aconselhamento sobre o tratamento e pacientes com melhor qualidade de vida. Uso anterior de drogas ilicitas em qualquer período da vida está associada à pior adesão. A adesão global foi baixa, enfatizando a necessidade de estratégias focadas no aconselhamento sobre medicamentos e uso de drogas. A quantidade de comprimidos não foi um problema para pacientes em uso de MTR-DTG uma vez ao dia, os quais alcançaram melhores taxas de adesão.

HIV; Antirretrovirais; Adesão à Medicação; Autorrelato

\section{Resumen}

Evaluamos la adherencia a la terapia antirretroviral altamente activa (TARAA) y sus factores asociados, según el tipo de tratamiento en pacientes que comenzaron su tratamiento en Belo Horizonte, Minas Gerais, Brasil. La adherencia se mensuró por la Escala de Adhesión Terapéutica de Morisky, de ocho ítems (MMAS-8), y se comparó el uso del "eje" tenofovir/lamivudina, además de un comprimido de efavirenz una vez al día (STR) o dolutegravir con varios comprimidos una vez al día (MTR-DTG), u otros tratamientos con múltiples comprimidos (MTR-otros). Se realizó un análisis multivariado de regresión logística para evaluar los factores asociados a la adherencia. Se incluyeron un total de 393 pacientes, 254 usaron STR, 106 MTR-DTG, y 33 MTR-Otros. La tasa de adherencia general fue de un 44,8\% (95\%CI: 39,4; 50,1), 50\% en el MTR-DTG, 43,3\% en el STR y 39,4\% en el MTR-otros. El análisis multivariado mostró una probabilidad más alta de adherencia entre pacientes usando MTR-DTG, quienes recibieron y comprendieron las orientaciones acerca de sus tratamientos y los que disfrutaban de una calidad mejor de vida. El consumo previo de drogas ilícitas a lo largo de la vida estuvo asociado con una adherencia más escasa. La adherencia general fue baja y resalta la necesidad de estrategias que se enfoquen en brindar orientación sobre el uso de la medicación y de sustancias. El número de comprimidos no fue un problema para los pacientes que tomaban MTR-DTG una vez al dia, que obtuvieron mejores resultados.

VIH; Antirretrovirales; Cumplimiento de la Medicación; Autoinforme
Submitted on $12 /$ Jun/2018

Final version resubmitted on 25/Jan/2019 Approved on 05/Apr/2019 